\title{
Cost-Effective Wire-Harness Model by Using Polymer Optical Fiber
}

\author{
Mohammad Syuhaimi Ab-Rahman and Farshad Nasimi Khameneh \\ Department of Electronic and System Engineering, \\ Faculty of Engineering and Built Environment, University Kebangsaan, Bangi, Malaysia
}

Received 2012-10-26, Revised 2012-12-18; Accepted 2013-06-25

\begin{abstract}
In the past decade automotive industries faced the exponential increase of in-vehicle electronic devices. The hydraulic systems are replacing with sophisticate electronic systems. Market demands for exploiting new in-vehicle technologies such as multimedia systems, internet access, GPS, Mobile communication, internal private network; engine, body and power train intelligent control and monitoring systems are increasing daily. These new needs make the wire-harness as physical pathway for power and data more complex. The amount of different data types' transmission in vehicle networking requires higher bandwidth and subsequently applying expensive and advanced equipment. Also more functions and facilities lead to raise the number of Electronic Control Units (ECU). The high cost of manufacturing and implementing all mentioned equipment and systems only can be justified to luxury vehicle's high prices. This study presents a conceptual model of in-vehicle networking which would lead to apply considerable portion of these advanced systems in non-luxury vehicles. In this context, Polymer Optical Fibers (POF) exploited to achieve high speed bandwidth and cost-effective solution to transfer huge amount of data and one ECU to control and manage body/cabin electronic devices. Regarding to technical specification of POFs and using visible light as data carrier, they can meet all new needs of implementing modern expected technologies for non-luxury cars at inexpensive solution. In addition, POFs are easy-to-use, reliable and flexible in compare with silica base optical fibers. This study suggests three red, blue and green lights for transferring video/audio, communication data network such as internet/vehicle internal network and body/cabin command lines respectively. Moreover, this concept model claims for reducing wire-harness with integration of command lines into multiplexed POF line. By command lines integration also it is possible to merge several power lines into one load calculated and shared power wire along the vehicle. After that, this conceptual model can decrease the amount of different connectors and control switches in car electronic systems. At last, this solution can effects on all steps of vehicle design, manufacturing and assembly process, especially in electronic section.
\end{abstract}

Keywords: Wire Harness, In-Vehicle Network, Polymer Fiber Optic (POF), Electronic Control Unit (ECU)

\section{INTRODUCTION}

These days, over $30 \%$ of total luxury cars cost is electronic systems and this magnitude is increasing steadily (Mahfoud et al., 2008). Daily development in computer and electronic technologies push automotive manufacturers ahead to invent and fit several facilities and features to their products. The increase of electronic devices in vehicle (car, bus, train and airplane) means more power supply and communication infrastructures and followed by that increasing of fuel consumption (Kassakian et al., 1996). In early of 1960s, when there was any discussion about automotive electrical systems it led to single 12 volte

Corresponding Author: Mohammad Syuhaimi Ab-Rahman, Department of Electronic and System Engineering,

Faculty of Engineering and Built Environment, University Kebangsaan, Bangi, Malaysia 
liquid acid battery, alternator and simple Wire-harness for support power loads. Wire-harness was just a bunch of copper wires for distributing power through vehicle. The 1980 decade was the big bright spot of applying electronic systems in automotive history and each manufacturer started to introduce its own predomination of new features (Leen et al., 1999) and also this time was the golden age of computer industry revolution. First generation of computer embedded systems in vehicles came to exist and it was huge evolutionary step in high speed data exchange and processing for improving control and monitoring methods. Semiconductors made it possible to produce more cost-effective and smaller electronic components and then reducing cost of design, production and manufacturing process, installation and implementing, man power and at last power load. Wireless and Bluetooth technology became a vital part of shortdistance of in-vehicle networking to improve telematics communication. Now talking about vehicle electronic systems is about different protocols and communication standards with complex power supply and distribution on various voltages levels. By coming Multimedia and informatics systems in the vehicle system requesting for high bandwidth raised hence the Wire-harness and internal multiplex network become more complicate and highly cost. Some automotive companies like BMW, Mercedes Benz, Chrysler, Audi and GM have used fiber optic technology to upgrade data transmission speed and modify their wire harness, but no work has done for make cost-effective solution for non-luxury cars. In recent years, these companies are cooperating for exploiting new standard so-called Media Oriented Systems Transport (MOST) to respond bandwidth hungry in-vehicle networking. Basically, using fiber optic as the main in-vehicle data bus is the core solution of the MOST technology (Seibl et al., 2008). The purpose of this study is to discuss, the wiring reduction in the car by using Polymer Optic Fiber (POF) as data transmission medium and merging power lines with consider of distribution system. Wire-harness section describes the characteristics of today vehicle Wiring regarding of its roles as power distributer and data transmission in the vehicle. Section POF as communication medium describes POF as ideal data transmission in physical pathway level. Section state-of-the-art presents new concept of Wire-harness which it is the main approach of this study and at last the concluding of the study.

\subsection{Automotive Facilities and Features}

Three important factors became to core attention of all automotive companies to improve their products. These three are safety, security and comfort in manner of considering maximum performance with competitive price. To aim to more comfort, safety and security with high expected performance over 100 control module units or Electronic Control Unit (ECU), several hundred sensors/actuators and Hi-tech multiplex communication networks with approximately 2500 signal per second have been employed in expensive cars (Worroll, 2011). Of course implementing of expensive equipment is not reasonable for all class of vehicles hence automotive companies categorized the usage of electronic systems according these three factors due to customers' budget. Obviously this matter became a limitation for implementing some facilities like navigation systems, entertainment equipment, cabin comfort systems, smart diagnosis systems and internet access to low-range price automobiles. The two of the most important barriers on this way are providing suitable communication medium and power supply infrastructure with minimum complexity and cost in which both of them are subjected to Wire-harness design technology.

\subsection{Wire Harness}

These days we cannot explain and evaluate wiring system in vehicle without focusing on ECUs and electronic devices. Du to increase of applying new features, the Wire-harness itself became expensive part of vehicle. Diversity of electronic devices needs different voltage for power and various network infrastructures for exchanging their output and input data with other elements. To the best level of vehicle operation all ECUs must be interconnected to each other, for example ABS's ECU must have information of engine torque data, speed of vehicle and active suspension to the best action. Even some comfort and auxiliary facilities which handle with some microcontrollers have interconnection with invehicle network (Shilbayeh and Iskandarani, 2005). Hence it is necessary to have two communication channels for each ECU. In order to have $\mathrm{n}$ ECUs, the network need to provide $\mathrm{n}^{2}$ communication channels and at the result a bulky and heavy point-to-point wire harness. Nowadays, $18 \%$ of entire car weight is electronic systems and it is expected to reach to $50 \%$ in the near future. The Wireharness weight after engine and gear-box is about $10 \%$ of total vehicle weight and in the third level of vehicle parts weight (Barmada et al., 2010). In the middle of 
1990 sec, automotive suppliers companies introduced Controller Area Network (CAN) to overcome the problem of daily expanded Wire-harness (Kimm et al., 2009). After that, many other protocols came to exist in response to meet new electric feature of vehicle which they are not in the scope of this study. Basically, for evaluation of Wire-harness it needs to consider from three points of view; connectors, power distribution line and data communication medium.

\subsection{Connectors}

At first glance may be connectors and their properties do not look very important but when the scale of design become as small as one or two square centimeter and any fault in connections can cause of major injury or death (fire or accident); everything will be crucial. In a normal home-use vehicle because of diversity of devices like variety of sensors, actuators, electronic device and different power supply ports (wire gauges), there are over of thirty kinds of connectors (Hult, 2012). The average number of connection points in the modern vehicle is 200 points. The design, material, size, weight and the way of locating in the vehicle space make serious difficulties for automotive manufacturers. Recently, using new technologies like GPS, video camera, video and audio streaming, cell phone, infrared night vision, internet and local network, advance cruise control and many others; provoke mentioned difficulties. Usually connectors provide by Wire-harness suppliers and considerable share cost is dedicated to them (Leen and Haffernan, 2002). Hence, finding new solutions to optimize connectors design without decreasing functionality is one of the important attentions of vehicle manufacturer. During past ten years, some of automotive companies and their suppliers attempted to standardize and improve the exploited connectors in the products. One of the best experimented and applied connectors was optic fiber ones which it come with fiber optic harness on the implementing entertainment in luxury cars (MOST technology) (Strobel et al., 2010). According to the MOST technology, these connectors are lighter, easy-to-install, safer, space conservation, smaller and because of optic fiber characteristic, they are immune to Electromagnetic Interferences (EMI) and support high bandwidth with maximum security. Up until 2008 the exploited optic fibers in the MOST technology was Glass type but MOST Company started to conduct some test and research for replacing Glass fiber optic (silicon fiber) with POF because of its low expenses and easier to use (Pardo, 2010). The development of POF infrastructure in automotive still is under the process. Now POF is used for infotainment data bus in some luxury cars like BMW, Mercedes Benz, Audi and other cooperatives of MOST company.

\subsection{Power Distribution}

The most important factor in the today world is fuel (oil, gasoline and gas) as main sources of energy. Many institutes and research centers in developed countries cost millions of dollars to reduce fuel consumption. In the vehicle, power generator and storage are alternator and battery respectively. Wire-harness is responsible for distributing power along the vehicle body and its parts. Figure 1 shows the traditional diagram of power distribution up until later of 1960s. As mentioned after introducing new features and rising up electronic systems portion in the vehicle; Wire-harness became more complicated. Thus, Wire-harness in addition to support power for devices it became medium of data transmission. Wires with different gauges exploited for several applications. Now over 900 signal/command wires are using in vehicle wiring and length of modern Wire-harness is over several kilometers. In term of battery and alternator many research and successful experiment has been done but still there is more room for improving wiring design.

For example some manufacturers came up with idea of 42 volt electrical power for vehicles in replace of traditional 12 volt ones. The reason of this working voltage changing is reducing ohmic losses without any major changes in wire harness. The calculation of energy wasting though heat, moving parts of engine and load devices is out of this study scope but the effects of weight and electrical resistance of Wire-harness are the main considerations (Ullah et al., 2012). In case of weight, Sumitomo Group produced new lighter and cables with aluminium (for low voltage application) for Toyota Motor in japan about $20 \mathrm{~kg}$ lighter than copper one (Sumitomo, 2010). Another example is Mercedes Sclass which reduced $50 \mathrm{~kg}$ from entire of vehicle weight by using POF replacing copper (Daishing, 2011). Refer to classical mechanic science, which reducing the amount of weight is directly proportional to fuel consumption and this magnitude is approachable by comparing power-to-weight ratio in different vehicle models. According to statistics by reducing about $45 \mathrm{~kg}$ of the entire weight of vehicle we can save up to $3.8 \%$ $\mathrm{KM} / \mathrm{L}$ (EEA, 2001). Moreover with improving efficiency of electrical systems (choosing appropriate devices and wire gauges) it is possible to cut off 100 watt of average load and it is same as reducing $50 \mathrm{~kg}$ of car's weight in fuel economical calculation. 

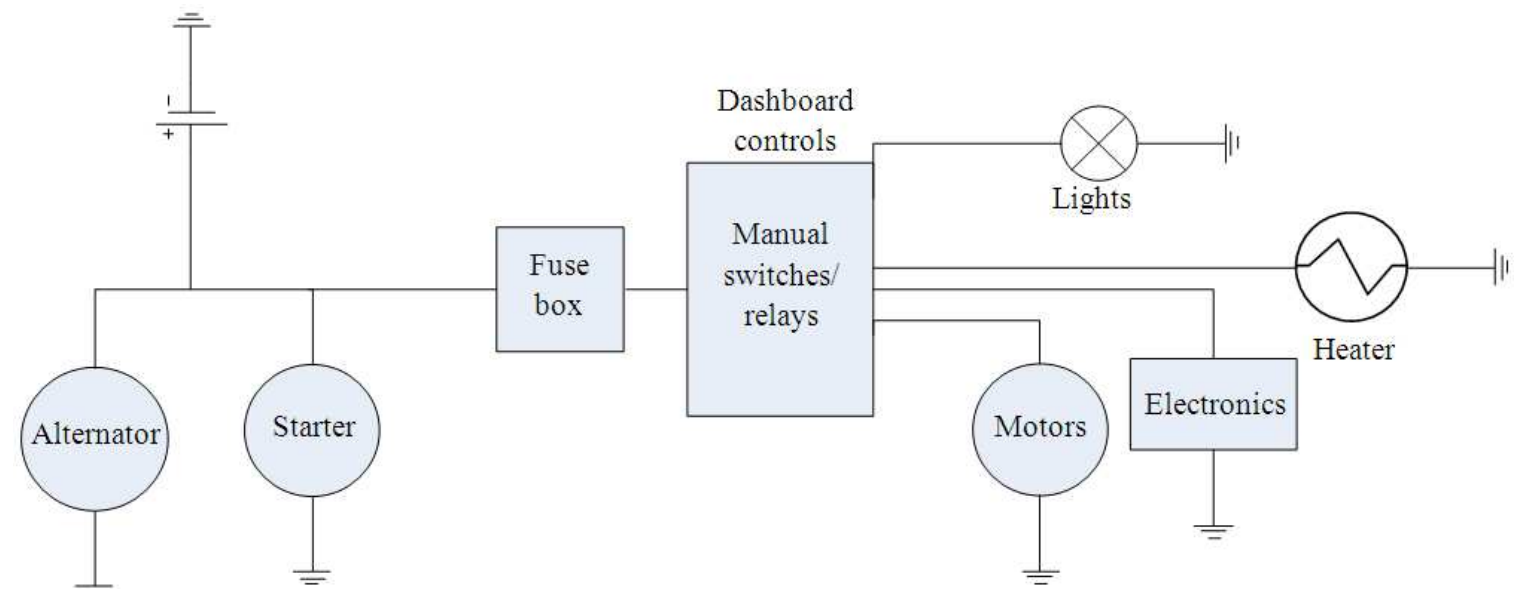

Fig. 1. Traditional Diagram of power distribution

Table 1. Characteristics of POF

\begin{tabular}{lll}
\hline Characteristic & Unit & Magnitude \\
\hline Core diameter & $\mu \mathrm{m}$ & 980 \\
Cladding diameter & $\mu \mathrm{m}$ & 1000 \\
Reflective index profile & & Step-index \\
Numerical aperture & & 0.5 \\
Attenuation between 630 to 650 & $\mathrm{~dB} / \mathrm{m}$ & $<0.4$ \\
Diameter of outer jacket & $\mu \mathrm{m}$ & 2300 \\
Operation temperature & ${ }^{\circ} \mathrm{C}$ & -40 to +85 \\
Minimum bending radius & $\mathrm{Mm}$ & 25 \\
Inner and outer jacket & & Polyamide \\
Tensile strength & $\mathrm{N}$ & 100 \\
Elongation at $100 \mathrm{~N}$ & $\%$ & $<2.5$ \\
\hline
\end{tabular}

\subsection{Data Communication Medium}

The simple model of data communication in the vehicle is consist of three parts; actuator, controller (can be an ECU) and actuator (Nolte et al., 2005). Normally in non-luxury and middle range price vehicles there are 1 to 3 ECUs. The number of ECUs presents the complexity of the both physical and application layer of in-vehicle networking. Technically, the communication in the modern car's network is consist of 6 functional domain groups; (1) power train (2) chassis domain (3) body domain (4) informatics, telematics and HMI (5) Active/Passive safety (6) Diagnostic (Mahmud and Sheran, 2005). However in non-luxury vehicles because of high-end cost of products implementing of all 6 mentioned functions is impossible and manufacturers try to combine first two groups into one and one extra for handling body electronics sell their product; totally with two ECUs. Still informatics function enumerates as the luxury facility in the majority of today's automobiles. As discussed before, one of the important factors which have direct effect on automobile price is electronic systems, especially power supply and data exchange infrastructure in which Wire-harness is the main part of both of them. There are 19 well-known automotive networks which fiber optic is used in 3 of them, 10 of them used Twisted pair, 3of them are normal 2wire (untwisted) and the rest are normal single wire (Navet and Simonot-Lion, 2009). All optic fiber based networks usually used for video and audio streaming because they provide high bandwidth channel connections and the rest used for controlling purpose. Every network must meet 4 factors to satisfy modern aspects of up-to-date communication design standards; first is the price factor which is the vital factor for wild commutative automotive market these days, second is immunity from EMI which usually shields handle this item for copper based cables and optic fibers are totally immune, third is adaptability to in-vehicle environments and fourth factor is robustness and amount of occupied space in the vehicle (Ramesh and Murthy, 2005). According to SAE network speed categorizing there are 4 classes of multiplexing networks; low speed, medium speed, high speed and very high speed which they are below 10 $\mathrm{Kbit} / \mathrm{sec}, 10 \mathrm{~Kb} / \mathrm{s}$ to $125 \mathrm{~Kb} / \mathrm{s}, 125 \mathrm{~Kb} / \mathrm{s}$ to $1 \mathrm{Mb} / \mathrm{s}$ and greater than $1 \mathrm{Mb} / \mathrm{s}$ respectively (Lupini, 2010). Up until now manufacturers believed that implementing even polymer optic fibers infrastructure for in-vehicle networks is became costly for their products they did not intend to apply it (except MOST technology that exploited for informatics and multimedia purpose in the expensive automobiles). The main purpose of this study beside of power distribution model suggestion is using POF instead of twisted pair cables as well. For this approach, authors exploited self-fabricated cost-effective WDM multiplexer to reach maximum bandwidth. 


\subsection{POF as Communication Medium}

Polymethylemthacrylat fiber (PMMA), so-called Polymer Optical Fiber (POF) is the strong, low cost and lightweight fiber. POF can transmit $92 \%$ of visible light and pass infrared light up to $2800 \mathrm{~nm}$. Table 1 shows the characteristics of POF. Due to large core diameter it is easy for coupling, quick installing and maintaining and more prone to scratching in compared to conventional inorganic glass (Ziemann et al., 2008). A LightEmitting Diode (LED) is used for transmitting data and Si-photodiode as receiver; they convert electrical signal to optical and vice versa respectively. Today many of POF-base devices became commercial for short distance video and audio transmission. The speed of data transmission in step-index type is 400 $\mathrm{Mb} / \mathrm{s}$ below $100 \mathrm{~m}$ (Lopez et al., 2009).

\subsection{Wavelength Division Multiplexing}

The capability of multiplexing (join) different wavelengths (optical carrier) into single POF on sender side and splitting them back on receiver side is the critical advantage of its operation in vehicle networking (Union, 2003). WDMs expand the capacity of networks without using extra fibers. The MOST company introduced high performance bidirectional WDM called LISW for high speed transmission with using red and green wavelength (2010). A fusion-based multiplexer and filtering-based splitter (de-multiplexing) was successfully fabricated in the UKM University. Figure 2 shows the filter base fabricated POF splitter/coupler structure. The multiplexer (coupler) and splitter devices were successfully applied for transferring Ethernet (data), video and audio by three wavelengths of $470 \mathrm{~nm}$ (blue), $510 \mathrm{~nm}$ (green) and $665 \mathrm{~nm}$ (red) respectively (Ab-Rahman et al., 2009b).

Figure 3 presents the attenuation results of transmission of audio, video and data through the POF in the given distances. The result reveals using three different color codes (wavelengths) to transfer data on POF is possible but in short distances. As Fig. 3 shows, below of $5 \mathrm{~m}$ the power loss magnitude is acceptable; although simultaneously injection of three colors caused the decreasing of red light power in POF but even until 10 (less than-22 db) meter is reasonable.

Regarding to the low rate of the sensors/ECU/actuators data communications; the power loss at $5 \mathrm{~m}$, is suitable for the in-vehicle networking usage. Authors used POF with WDM in the suggested concept for add up the capability of control and monitoring features without extra lying fibers in the vehicle. Basically, between $480 \mathrm{~nm}$ to $530 \mathrm{~nm}$ there is large attenuation area and it cause of considerably drop of speed of data transmission but for compensation of wavelength weakness some high speed LEDs is used. Most cooperation GaN LEDs to rates up data transmission speed to $500 \mathrm{Mb} / \mathrm{sec}$ with green wavelength (Ab-Rahman et al., 2009a). It must noticed that there are other method like using band-pass filter and prism to multiplexer/de-multiplexer but not cost-effective.

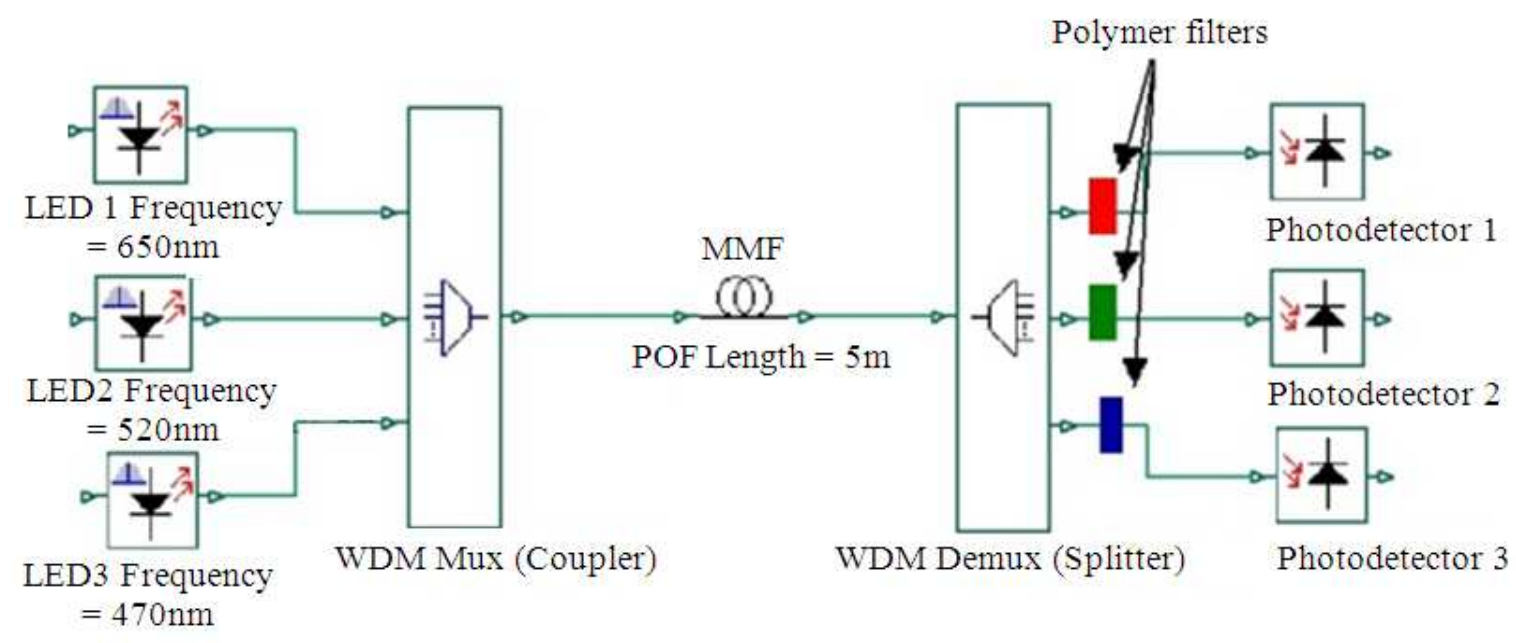

Fig. 2. POF-WDM filter base structure 
M.S. Ab-Rahman and F.N. Khameneh / Journal of Computer Science 9 (7): 935-942, 2013

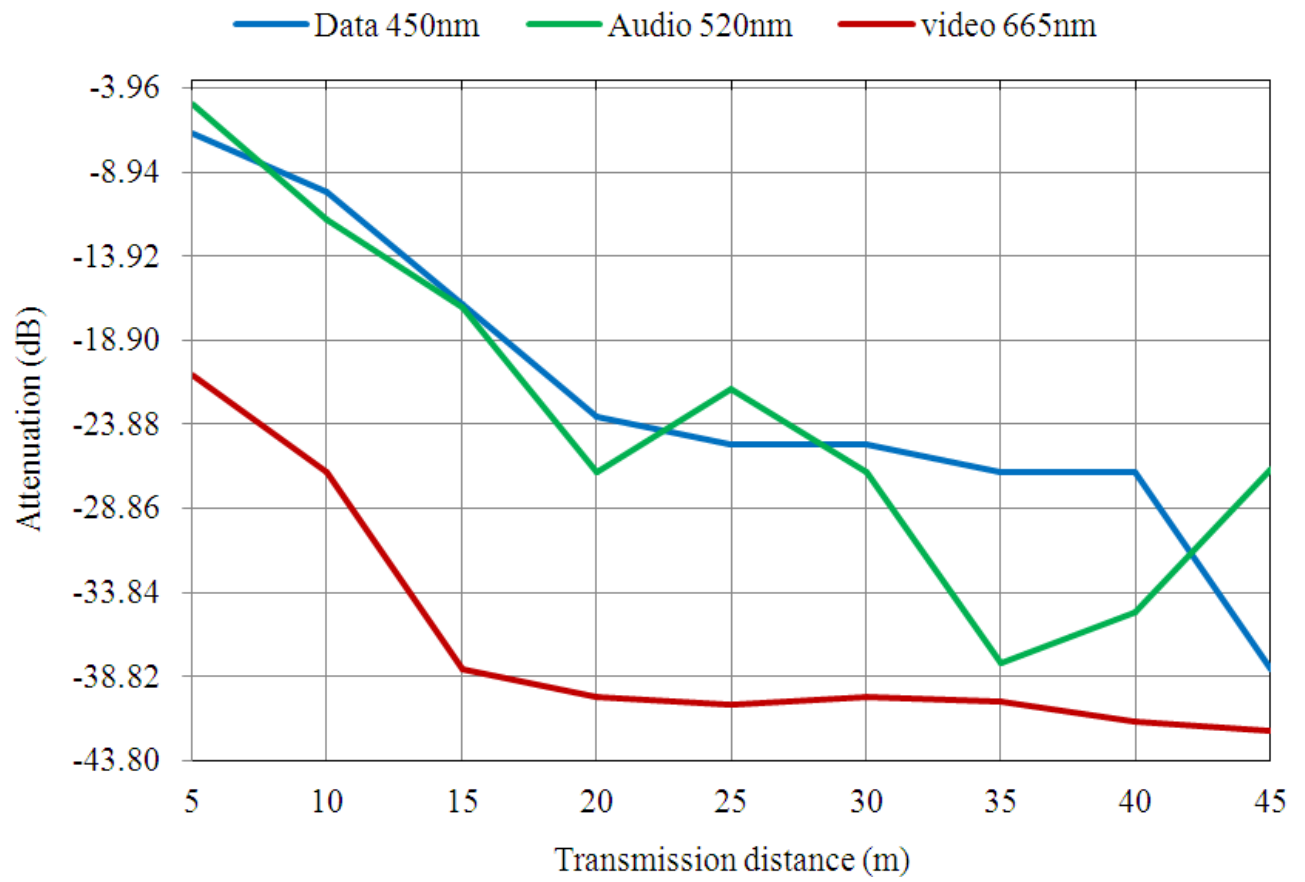

Fig. 3. POF-WDM Transmission losses on distance

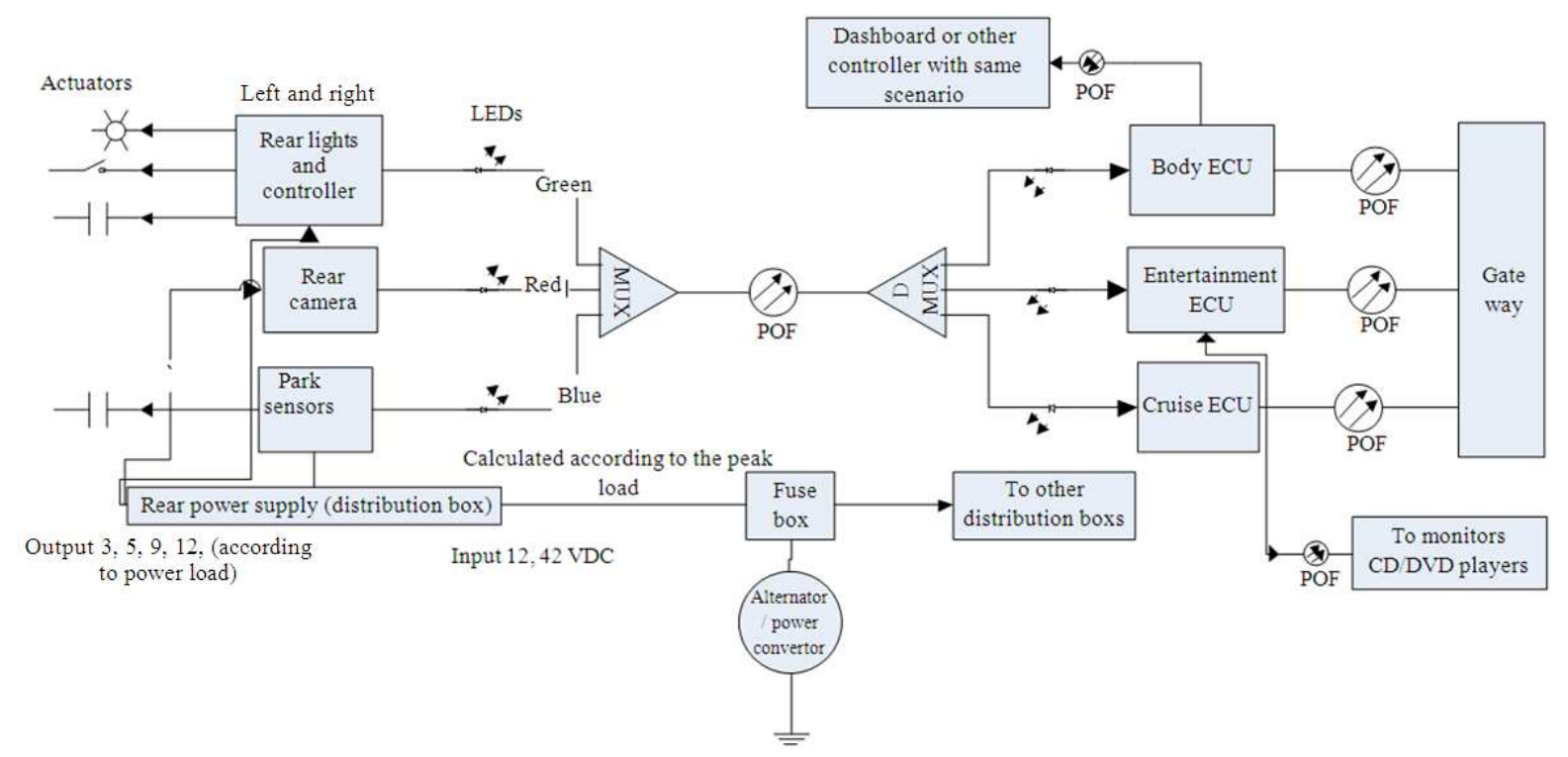

Fig. 4. Conceptual model of wire-harness

\subsection{State-Of-The-Art}

According to what discussed in pervious sections, here the new conceptual model of Wire-harness is presented. Mainly the approach of paper content is based on 42 volt power systems and using POF as main data transmission medium in the vehicle. Definitely it is possible to use this conceptual model for $12 \mathrm{v}$ power systems but the number of power supply will be increased. Some points are necessary to be considered. 
First, this concept shows the possible combination of copper cable as power line back bone and POF with using WDM for data transferring Just in physical layer. The main reason of using POF even instead of twisted wires is the low cost of implementation of this kind of fiber equipment (cable, transmitter, receiver, connector and WDM) notwithstanding of knowing the capacity of POF data transmission is over ratio of what needed to transfer sensor and actuators data. It is possible to use software technologies for exploiting this remain portion of bandwidth. The suggested topology for power distribution is star but for data network if there is more than 3 ECU in the network design, can be combination of star (between ECUs and subset controllers) and ring (between ECUs themselves-like as MOST plan); if the amount of ECUs $>=3$ then the suggested topology is the star with one gateway. The main consider of power distribution is the location of load nodes. The shared powers among of several power loads are not calculated because it is different from one car to another. As the Fig. 4 shows, the suggested model is illustrated on the rear vehicle facilities and the scenario is same for other parts of vehicle.

For all controllers, one extra POF cable with same wavelength has considered conducting bidirectional channel (not shown in the diagram). It means between Rear light controller and body ECU there is another POF to provide return data channel from body ECU to the Rear Light controller with same colour (here is green)but on different MUX/D-MUX. The reason of choosing same colour code by authors is considering about integrity of colours usage with relation of device on same channels. Also every chosen wavelength is on the base of required device bandwidth. For example Rear camera needs more bandwidth, thus the red wavelength which support more data transmission speed allocated on. Another good example of ready to implementation wiring is the integration of 4-park sensor (equipped with camera) with rear light controller into the 2 bidirectional couple/splitter POF data transmitting lines.

\section{CONCLUSION}

This study suggests a conceptual model of in-vehicle network in both power supply and data communication to follow the purpose of reducing Wire-harness as one of the significant automotive manufacturer problems. Since these days about $90 \%$ of exploited wires in the vehicle are copper base and it caused the Wire-harness became expensive, heavy, bulky and complex; the suggestion of this model to reduce that (in both weight and size) is using
POF even instead of twisted and twisted pair cables. Basically, because of characteristics of POF and capability of WDM on POF, this model can be compatible to Truck, bus, boat and small airplane's networks.

\section{REFERENCES}

Ab-Rahman, M.S., H. Guna and M.H. Harun, 2009a. Realization of an economical $1 \times 3$ fused-taper-twisted polymer optical fiber splitters for small world communication. Malaysian J. Sci., 28: 289-297.

Ab-Rahman, M.S., M.H. Harun and H. Guna, 2009b. Low-cost cascaded $1 \times 4$ polymer optical fiber coupler for multiplexing wavelengths. Proceedings of the 9th Malaysia International Conference, Dec. 15-17, IEEE Xplore Press, Kuala Lumpur, pp: 5559. DOI: 10.1109/MICC.2009.5431401

EEA, 2001. Owener Related Fuel economy improvements. Oak Ridge National Laboratory.

Barmada, S., M. Raugi, M. Tucci and T. Zheng, 2010. Power line communication in a full electric vehicle: Measurements, modelling and analysis. Proceedings of the IEEE International Symposium on Power Line Communications and Its Applications, Mar. 28-31, IEEE Xplore Press, Rio de Janeiro, pp: 331336. DOI: 10.1109/ISPLC.2010.5479920

Daishing, J., 2011. The application of POF in automobile industry. Jiangxi DaIshing POF Co., Ltd.

Hult, B., 2012. Evolving Automotive Connectors.

Kassakian, J.G., H.C. Wolf, J.M. Miller and C.J. Hurton, 1996. Automotive electrical systems circa 2005. IEEE Spectrum, 33: 22-27. DOI: 10.1109/6.511737

Kimm, H., S. Shin, H. Ham and C.O. Sung, 2009. Failure management development for integrated automotive safety-critical software systems. Proceedings of the ACM Symposium on Applied Computing, Mar. 9-12, ACM Press, New York, USA., pp: 517-521. DOI: 10.1145/1529282.1529390

Leen, G. and D. Haffernan, 2002. Expanding automotive electronic systems. IEEE Comput., 35: 88-93. DOI: $10.1109 / 2.976923$

Leen, G., D. Heffernan and A. Dunne, 1999. Digital networks in the automotive vehicle. Comput. Control Eng. J., 10: 257-266. DOI: 10.1049/cce:19990604

Lopez, C., D.F. Nespola, A. Camatel, S. Abrate and S.R. Gaudino, 2009. $100 \mathrm{Mb} / \mathrm{s}$ ethernet transmission over $275 \mathrm{~m}$ of large core step index polymer optical fiber: Results From the POF-ALL European project. J. IEEE Lightwave Technol., 27: 2908-2915. DOI: 10.1109/JLT.2009.2019609 
Lupini, C.A., 2010. In-vehicle networking technology for 2010 and beyond. SAE Int., 1: 1-14. DOI: 10.4271/2010-01-0687

Mahfoud, M., N. AL-Holou and R. Baroody, 2008. Next generation vehicle network: Web enabled. Proceedings of the 3rd International Conference on Information and Communication Technologies: From Theory to Applications, Apr. 7-11, IEEE Xplore Press, Damascus, pp: 1-7. DOI: 10.1109/ICTTA.2008.4529964

Mahmud, S.M. and A. Sheran, 2005. In-vehicle network architecture for the next-generation vehicles. Wayne State University.

Navet, N. and F. Simonot-Lion, 2009. Automotive Embedded Systems Handbook. 1st Edn., Taylor and Francis Group, Boca Raton, ISBN-10: 084938026X, pp: 488.

Nolte, T., H. Hansson and L.L. Bello, 2005. Automotive communications-past, current and future. Proceedings of the IEEE Conference on Emerging Technologies and Factory Automation, Sept. 19-22, IEEE Xplore Press, Catania, pp: 992-999. DOI: 10.1109/ETFA.2005.1612631

Pardo, C., 2010. Application of advanced communication techniques within KDPOF physical layer, to achieve a Robust and Low-Cost MOST100. Proceedings of the 12th International Conference on Transparent Optical Networks, Jun. 27-Jul. 1, IEEE Xplore Press, Munich, pp: 1-4. DOI: 10.1109/ICTON.2010.5549162

Ramesh, B.K. and S.K. Murthy, 2005. In-vehicle networking. Dearborn Electron.
Seibl, D., M. Bohm and O. Strobel, 2008. PolymerOptical-Fiber data bus technologies for MOST applications in vehicles. Proceedings of the 2nd ICTON Mediterranean Winter, Dec. 11-13, IEEE Xplore Press, Marrakech, pp: 1-6. DOI: 10.1109/ICTONMW.2008.4773071

Shilbayeh, N.F. and M.Z. Iskandarani, 2005. Wireless control of vehicle mirror system using head movement and pic microcontroller. J. Comput. Sci., 1: 182-188. DOI: $10.3844 /$ jcssp.2005.182.188

Strobel, O., R. Rejeb and J. Lubkoll, 2010. Communication in automotive systems: Principles, limits and new trends for vehicles, airplanes and vessels. Proceedings of the 12th International Conference on Transparent Optical Networks, Jun. 27-Jul. 1, IEEE Xplore Press, Munich, pp: 1-6. DOI: 10.1109/ICTON.2010.5549163

Sumitomo, E.I., 2010. Automotive low-voltage wiring harness using aluminum wires is used for new Ractis from Toyota Motor Corporation. Sumitomo Wiring Systems, Ltd.

Ullah, M.H., T.S. Gunawan, S.M. Raihan and R. Muhida, 2012. Development of a electrically inspired low emission microcontroller based hybrid vehicle. Am. J. Sci., 9: 1729-1735.

Union, I.T., 2003. Transmission systems and media, digital systems and networks.

Worroll, T., 2011. Automotive connectors evolve to meet complex demands and EV challenges. ITT Interconnect Solutions.

Ziemann, O., J. Krauser, P.E. Zamzow and W. Daum, 2008. POF Handbook: Optical Short Range Transmission Systems. 2nd Edn., Springer, Berlin, ISBN-10: 3540766286, pp: 884. 